\title{
Calculation of transverse piezoelectric characteristics of quasi-one-dimensional glycine phosphite ferroelectric
}

\author{
Vdovych A. ${ }^{1}$, Zachek I. ${ }^{2}$, Levitskii R. ${ }^{1}$ \\ ${ }^{1}$ Institute for Condensed Matter Physics of the National Academy of Sciences of Ukraine, \\ 1 Svientsitskii Str., Lviv, 79011, Ukraine \\ ${ }^{2}$ Lviv Polytechnic National University, 12 S. Bandera Str., Lviv, 79013, Ukraine
}

(Received 3 December 2018)

\begin{abstract}
The model of the glycine phosphite crystal, modified by taking into account of piezoelectric coupling of ordering structure elements with the lattice strains, is used for investigation of piezoelectric characteristics of the crystal. In the frames of two-particle cluster approximation the transverse piezoelectric coefficients are calculated. The influences of hydrostatic, uniaxial pressures, shear stresses and transverse electric fields on the transverse piezoelectric coefficients of the crystal are studied.
\end{abstract}

Keywords: ferroelectrics, phase transition, piezoelectric coefficients, pressure effects, external field effects.

2000 MSC: $82 \mathrm{D} 45,82 \mathrm{~B} 20$

UDC: $536.96,537.226(4,82,83,86)$

DOI: $10.23939 / \mathrm{mmc} 2018.02 .242$

\section{Introduction}

Investigations of the effects appearing under mechanical stress or external electric field is one of actual problems of physics of ferroelectric materials, especially for glycine phosphite crystal (GPI), that belongs to the ferroelectric compounds with hydrogen bonds [1].

On the basis of the proton model [2], in [3] there was created the model of deformed GPI crystal, which takes into account the piezoelectric coupling between proton and lattice subsystems. In [3] the components of polarization vector, dielectric permittivity tensor, longitudinal piezoelectric coefficients, elastic constants and molar heat capacity were calculated; a satisfactory description of the experimental data is obtained. The model of deformed GPI crystal made it possible to describe correctly the influence of transverse fields $E_{x} E_{z}$ [6], hydrostatic pressure [4] and uniaxial pressures [5] on these characteristics.

However, the symmetry of strains $\varepsilon_{4}, \varepsilon_{6}$ was not taken into account correctly in [3] and the transverse piezoelectric coefficients $e_{1 j}, e_{3 j}, h_{1 j}$ and $h_{3 j}$ were not calculated. In [7] the GPI model [3] is modified for the case of decreasing of symmetry under shear stresses $\sigma_{y z}$ and $\sigma_{x y}$. It was ascertained that the components of polarization vector $P_{x}$ and $P_{z}$ appear under the shear stresses $\sigma_{y z}$ and $\sigma_{x y}$, and the transverse permittivities $\varepsilon_{x x}$ and $\varepsilon_{z z}$ diverge at $T_{c}$ point.

In the present paper using the developed in [7] model of deformed GPI crystal the effects of hydrostatic and uniaxial pressures, shear stresses and transverse electric fields on the transverse piezoelectric characteristics of this crystal are studied.

\section{Hamiltonian of the model}

The model [7] consider the system of protons in GPI, localised on O-H. . O bonds between phosphite groups $\mathrm{HPO}_{3}$, which form zigzag chains along crystallographic $c$-axis of the crystal (see Fig. 1). For better understanding of the model only phosphite groups are shown in the figure. Dipole moments $\boldsymbol{d}_{q f}(f=1, \ldots, 4)$ are ascribed to the protons on the bonds. In the ferroelectric phase the dipole 


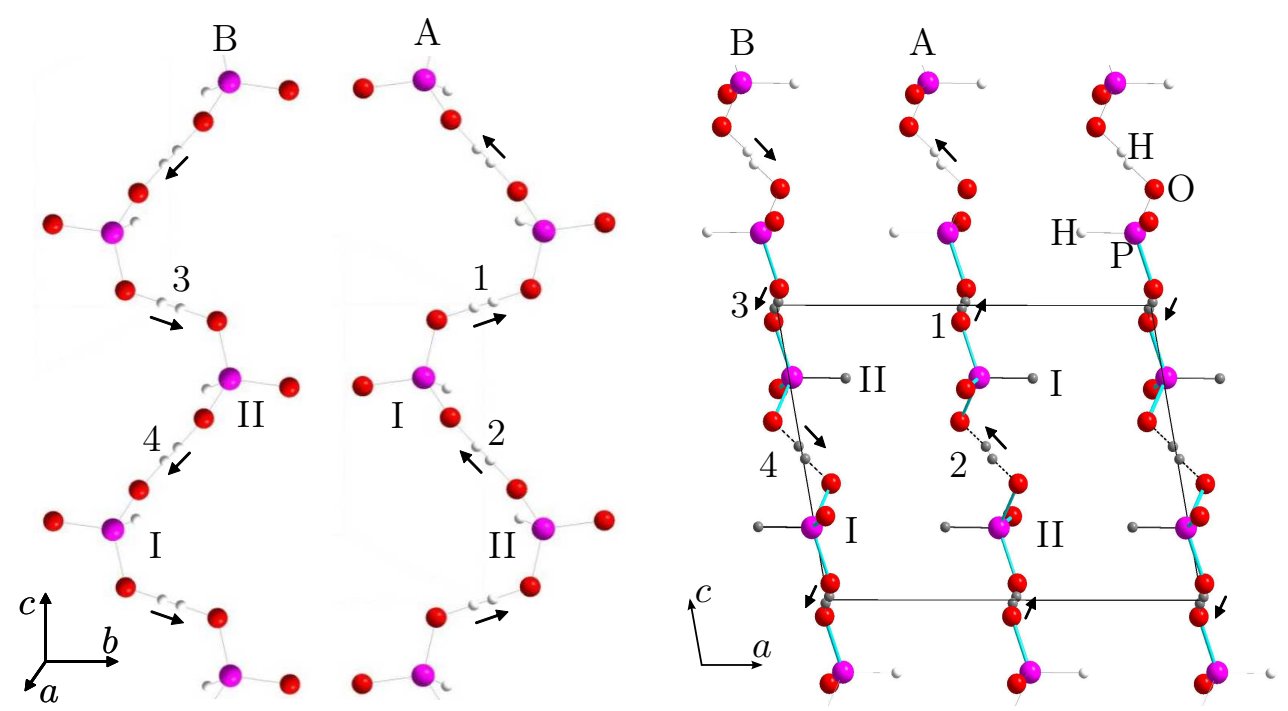

Fig. 1. Orientations of vectors $\boldsymbol{d}_{q f}$ in the primitive cell in the ferroelectric phase [3,7].

moments compensate each other $\left(\boldsymbol{d}_{q 1}\right.$ with $\boldsymbol{d}_{q 3}, \boldsymbol{d}_{q 2}$ with $\left.\boldsymbol{d}_{q 4}\right)$ in directions $\mathrm{Z}$ and $\mathrm{X}(\mathrm{X} \perp(b, c), \mathrm{Y} \| b$, $\mathrm{Z} \| c$ ), and simultaneously supplement each other in direction $\mathrm{Y}$, creating spontaneous polarization.

Pseudospin variables $\frac{\sigma_{q 1}}{2}, \ldots, \frac{\sigma_{q 4}}{2}$ describe reorientation of the dipole moments of the base units: $\boldsymbol{d}_{q f}=\boldsymbol{\mu}_{f} \frac{\sigma_{q f}}{2}$. Mean values $\left\langle\frac{\sigma}{2}\right\rangle=\frac{1}{2}\left(n_{a}-n_{b}\right)$ are connected with differences in occupancy of the two possible molecular positions, $n_{a}$ and $n_{b}$.

Below for the components of the vectors and tensors we use the notations 1, 2 and 3 instead of $x$, $y$ and $z$ for convenience. Hamiltonian of proton subsystem of GPI, which takes into account the shortrange and long-range interactions and the applied electric fields $E_{1}, E_{2}, E_{3}$ along positive directions of the Cartesian axes $\mathrm{X}, \mathrm{Y}$ and $\mathrm{Z}$ can be written in such a way [7]:

$$
\hat{H}=N U_{\text {seed }}+\hat{H}_{\text {short }}+\hat{H}_{\text {long }}+\hat{H}_{E},
$$

where $N$ is the total number of primitive cells. The term $U_{\text {seed }}$ in (1) is the "seed" energy, which relates to the heavy ion sublattice and do not depends explicitly on configuration of the proton subsystem. It includes the elastic, piezoelectric and dielectric parts, expressed in terms of electric fields $E_{i}(i=1,2,3)$ and strains $\varepsilon_{j}(j=1, \ldots, 6)$.

$$
U_{\text {seed }}=v\left(\frac{1}{2} \sum_{j, j^{\prime}=1}^{6} c_{j j^{\prime}}^{E 0}(T) \varepsilon_{j} \varepsilon_{j^{\prime}}-\sum_{i=1}^{3} \sum_{j=1}^{6} e_{i j}^{0} \varepsilon_{j} E_{i}-\sum_{i, i^{\prime}=1}^{3} \frac{1}{2} \chi_{i i^{\prime}}^{\varepsilon 0} E_{i} E_{i^{\prime}}\right) .
$$

Parameters $c_{j j^{\prime}}^{E 0}(T), e_{i j}^{0}, \chi_{i i^{\prime}}^{\varepsilon 0}$ are so called "seed" elastic constants, "seed" coefficients of piezoelectric stresses and "seed" dielectric susceptibilities, respectively; $v$ is volume of a primitive cell. Matrices $c_{j j^{\prime}}^{E 0}$, $e_{i j}^{0}, \chi_{i i^{\prime}}^{\varepsilon 0}$ are given by:

$$
\hat{c}_{j j^{\prime}}^{E 0}=\left(\begin{array}{cccccc}
c_{11}^{E 0} & c_{12}^{E 0} & c_{13}^{E 0} & 0 & c_{15}^{E 0} & 0 \\
c_{12}^{E 0} & c_{22}^{E 0} & c_{23}^{E 0} & 0 & c_{25}^{E 0} & 0 \\
c_{13}^{E 0} & c_{23}^{E 0} & c_{12}^{E 0} & 0 & c_{35}^{E 0} & 0 \\
0 & 0 & 0 & c_{44}^{E 0} & 0 & c_{46}^{E 0} \\
c_{15}^{E 0} & c_{25}^{E 0} & c_{35}^{E 0} & 0 & c_{55}^{E 0} & 0 \\
0 & 0 & 0 & c_{46}^{E 0} & 0 & c_{66}^{E 0}
\end{array}\right), \quad \hat{e}_{i j}^{0}=\left(\begin{array}{cccccc}
0 & 0 & 0 & e_{14}^{0} & 0 & e_{16}^{0} \\
e_{21}^{0} & e_{22}^{0} & e_{23}^{0} & 0 & e_{25}^{0} & 0 \\
0 & 0 & 0 & e_{34}^{0} & 0 & e_{36}^{0}
\end{array}\right),
$$

In the paraelectric phase all coefficients $e_{i j}^{0} \equiv 0$. 
Another terms in (1) describe the pseudospin part of hamiltonian. In particular, the second term in (1) is hamiltonian of short-range interactions:

$$
\hat{H}_{\text {short }}=-2 \sum_{q q^{\prime}}\left(w_{1} \frac{\sigma_{q 1}}{2} \frac{\sigma_{q 2}}{2}+w_{2} \frac{\sigma_{q 3}}{2} \frac{\sigma_{q 4}}{2}\right)\left(\delta_{\mathbf{R}_{q} \mathbf{R}_{q^{\prime}}}+\delta_{\mathbf{R}_{q}+\mathbf{R}_{\mathbf{c}}, \mathbf{R}_{q^{\prime}}}\right) .
$$

In (2) $\sigma_{q f}$ is the z-component of pseudospin operator, that describes the state of the $f$-th bond $(f=1,2,3,4)$, in the $q$-th cell. The first Kronecker delta corresponds to the interaction between protons in the chains near the tetrahedra $\mathrm{HPO}_{3}$ of type "I" (see Fig. 1), where the second one near the tetrahedra $\mathrm{HPO}_{3}$ of type "II", $\mathbf{R}_{\mathbf{c}}$ is the lattice vector along OZ-axis. Contributions into the energy of interactions between protons near tetrahedra of different type, as well as the mean values of the pseudospins $\eta_{f}=\left\langle\sigma_{q f}\right\rangle$, which are related to tetrahedra of different type, are identical.

Parameters $w_{1}, w_{2}$, which describe the short-range interactions within chains, are expanded linearly into series over strains $\varepsilon_{j}$ :

$$
\begin{aligned}
& w_{1}=w^{0}+\sum_{l} \delta_{l} \varepsilon_{l}+\delta_{4} \varepsilon_{4}+\delta_{6} \varepsilon_{6}, \quad(l=1,2,3,5) \\
& w_{2}=w^{0}+\sum_{l} \delta_{l} \varepsilon_{l}-\delta_{4} \varepsilon_{4}-\delta_{6} \varepsilon_{6} .
\end{aligned}
$$

The third term in (1) describes the long-range dipole-dipole interactions and indirect (through the lattice vibrations) interactions between protons, which are taken into account in mean field approximation:

$$
\hat{H}_{\text {long }}=\frac{1}{2} \sum_{\substack{q q^{\prime} \\ f f^{\prime}}} J_{f f^{\prime}}\left(q q^{\prime}\right) \frac{\left\langle\sigma_{q f}\right\rangle}{2} \frac{\left\langle\sigma_{q^{\prime} f^{\prime}}\right\rangle}{2}-\sum_{\substack{q q^{\prime} \\ f f^{\prime}}} J_{f f^{\prime}}\left(q q^{\prime}\right) \frac{\left\langle\sigma_{q^{\prime} f^{\prime}}\right\rangle}{2} \frac{\sigma_{q f}}{2} .
$$

Fourier transforms of interaction constants $J_{f f^{\prime}}=\sum_{q^{\prime}} J_{f f^{\prime}}\left(q q^{\prime}\right)$ at $\mathbf{k}=0$ are linearly expanded over the strains $\varepsilon_{j}$ :

$$
\begin{aligned}
& J_{\frac{11}{33}}=J_{11}^{0}+\sum_{l} \psi_{11 l} \varepsilon_{l} \pm \psi_{114} \varepsilon_{4} \pm \psi_{116} \varepsilon_{6}, \quad J_{13}=J_{13}^{0}+\sum_{l} \psi_{13 l} \varepsilon_{l}+\psi_{134} \varepsilon_{4}+\psi_{136} \varepsilon_{6}, \\
& J_{\frac{12}{34}}=J_{12}^{0}+\sum_{l} \psi_{12 l} \varepsilon_{l} \pm \psi_{124} \varepsilon_{4} \pm \psi_{126} \varepsilon_{6}, \quad J_{\frac{14}{23}}=J_{14}^{0}+\sum_{l} \psi_{14 l} \varepsilon_{l} \pm \psi_{144} \varepsilon_{4} \pm \psi_{146} \varepsilon_{6}, \\
& J_{\frac{22}{44}}=J_{22}^{0}+\sum_{l} \psi_{22 l} \varepsilon_{l} \pm \psi_{224} \varepsilon_{4} \pm \psi_{226} \varepsilon_{6}, \quad J_{24}=J_{24}^{0}+\sum_{l} \psi_{24 l} \varepsilon_{l}+\psi_{244} \varepsilon_{4}+\psi_{246} \varepsilon_{6} .
\end{aligned}
$$

In result, (3) can be written as:

$$
\hat{H}_{\text {long }}=N H^{0}-\sum_{q} \sum_{f=1}^{4} \mathcal{H}_{f} \frac{\sigma_{q f}}{2}
$$

where

$$
H^{0}=\sum_{f, f^{\prime}=1}^{4} \frac{1}{8} J_{f f^{\prime}} \eta_{f} \eta_{f^{\prime}}, \quad \mathcal{H}_{f}=\sum_{f^{\prime}=1}^{4} \frac{1}{2} J_{f f^{\prime}} \eta_{f^{\prime}} .
$$

The fourth term in (1) describes interactions of pseudospins with external electric field:

$$
\hat{H}_{E}=-\sum_{q f} \boldsymbol{\mu}_{f} \boldsymbol{E} \frac{\sigma_{q f}}{2} .
$$

Here $\boldsymbol{\mu}_{1}=\left(\mu_{13}^{x}, \mu_{13}^{y}, \mu_{13}^{z}\right), \boldsymbol{\mu}_{3}=\left(-\mu_{13}^{x}, \mu_{13}^{y},-\mu_{13}^{z}\right), \boldsymbol{\mu}_{2}=\left(-\mu_{24}^{x},-\mu_{24}^{y}, \mu_{24}^{z}\right), \boldsymbol{\mu}_{4}=\left(\mu_{24}^{x},-\mu_{24}^{y},-\mu_{24}^{z}\right)$ are the effective dipole moments per one pseudospin. 


\section{Piezoelectric characteristics of GPI}

Two-particle cluster approximation is used for calculation of thermodynamic characteristics of GPI. In this approximation thermodynamic potential per one primitive cell is given by [7]:

$$
\begin{aligned}
g=\frac{G}{N}= & U_{\text {seed }}+H^{0}-2\left(w^{0}+\sum_{l} \delta_{l} \varepsilon_{l}\right)+2 k_{\mathrm{B}} T \ln 2-N v \sum_{j=1}^{6} \sigma_{j} \varepsilon_{j} \\
& -\frac{1}{2} k_{\mathrm{B}} T \sum_{f=1}^{4} \ln \left(1-\eta_{f}^{2}\right)-2 k_{\mathrm{B}} T \ln D .
\end{aligned}
$$

where $\beta=\frac{1}{k_{\mathrm{B}} T}, k_{\mathrm{B}}$ is the Boltzmann constant. Here, such notations are used:

$$
\begin{aligned}
D= & \cosh n_{1}+\cosh n_{2}+a^{2} \cosh n_{3}+a^{2} \cosh n_{4} \\
& +a a_{46} \cosh n_{5}+\frac{a}{a_{46}} \cosh n_{6}+a a_{46} \cosh n_{7}+\frac{a}{a_{46}} \cosh n_{8}, \\
n_{1}= & \frac{1}{2}\left(y_{1}+y_{2}+y_{3}+y_{4}\right), \quad n_{2}=\frac{1}{2}\left(y_{1}+y_{2}-y_{3}-y_{4}\right), \quad n_{3}=\frac{1}{2}\left(y_{1}-y_{2}+y_{3}-y_{4}\right), \\
n_{4}= & \frac{1}{2}\left(y_{1}-y_{2}-y_{3}+y_{4}\right), \quad n_{5}=\frac{1}{2}\left(y_{1}-y_{2}+y_{3}+y_{4}\right), \quad n_{6}=\frac{1}{2}\left(y_{1}+y_{2}+y_{3}-y_{4}\right), \\
n_{7}= & \frac{1}{2}\left(-y_{1}+y_{2}+y_{3}+y_{4}\right), \quad n_{8}=\frac{1}{2}\left(y_{1}+y_{2}-y_{3}+y_{4}\right) . \\
y_{f}= & \frac{1}{2} \ln \frac{1+\eta_{f}}{1-\eta_{f}}+\frac{\beta}{2} \sum_{f^{\prime}=1}^{4} \frac{1}{2} J_{f f^{\prime}} \eta_{f^{\prime}}+\frac{\beta}{2} \boldsymbol{\mu}_{f} \boldsymbol{E}, \quad a=e^{-\beta\left(w^{0}+\sum_{l} \delta_{i} \varepsilon_{i}\right)}, \quad a_{46}=e^{-\beta\left(\delta_{4} \varepsilon_{4}+\delta_{6} \varepsilon_{6}\right)} .
\end{aligned}
$$

Minimizing the thermodynamic potential the system of equations for the strains $\varepsilon_{j}$ and the order parameters $\eta_{f}$, are obtained. Differentiating the thermodynamic potential over the fields $E_{1}$ and $E_{3}$ we obtain the expressions for polarizations $P_{1}$ and $P_{3}$ [7]:

$$
\begin{aligned}
& P_{1}=e_{14}^{0} \varepsilon_{4}+e_{16}^{0} \varepsilon_{6}+\chi_{11}^{\varepsilon 0} E_{1}+\frac{1}{2 v}\left[\mu_{13}^{x}\left(\eta_{1}-\eta_{3}\right)-\mu_{24}^{x}\left(\eta_{2}-\eta_{4}\right)\right] \\
& P_{3}=e_{34}^{0} \varepsilon_{4}+e_{66}^{0} \varepsilon_{6}+\chi_{33}^{\varepsilon 0} E_{3}+\frac{1}{2 v}\left[\mu_{13}^{z}\left(\eta_{1}-\eta_{3}\right)+\mu_{24}^{z}\left(\eta_{2}-\eta_{4}\right)\right]
\end{aligned}
$$

Differentiating expressions (8) over strains $\varepsilon_{1 j}$ the expressions for isothermic coefficients of piezoelectric stress $e_{1 j}, e_{3 j}$ of GPI are obtained:

$$
\begin{aligned}
& e_{1 j}=\left(\frac{\partial P_{1}}{\partial \varepsilon_{l}}\right)_{E_{1}}=e_{2 j}^{0}+\frac{1}{2 v}\left[\mu_{13}^{x}\left(\dot{\eta}_{1 \varepsilon_{j}}-\dot{\eta}_{3 \varepsilon_{j}}\right)-\mu_{24}^{x}\left(\dot{\eta}_{2 \varepsilon_{j}}-\dot{\eta}_{4 \varepsilon_{j}}\right)\right], \quad(j=4,6), \\
& e_{3 j}=\left(\frac{\partial P_{3}}{\partial \varepsilon_{j}}\right)_{E_{3}}=e_{3 j}^{0}+\frac{1}{2 v}\left[\mu_{13}^{z}\left(\dot{\eta}_{1 \varepsilon_{j}}-\dot{\eta}_{3 \varepsilon_{j}}\right)+\mu_{24}^{z}\left(\dot{\eta}_{2 \varepsilon_{j}}-\dot{\eta}_{4 \varepsilon_{j}}\right), \quad(j=4,6) .\right.
\end{aligned}
$$

where $\dot{\eta}_{1 \varepsilon_{l}}, \dot{\eta}_{2 \varepsilon_{l}}, \dot{\eta}_{3 \varepsilon_{l}}, \dot{\eta}_{4 \varepsilon_{l}}$ are the solutions of the following system of equations:

$$
\left(\begin{array}{cccc}
2 D-\varkappa_{11} & -\varkappa_{12} & -\varkappa_{13} & -\varkappa_{14} \\
-\varkappa_{21} & 2 D-\varkappa_{22} & -\varkappa_{23} & -\varkappa_{24} \\
-\varkappa_{31} & -\varkappa_{32} & 2 D-\varkappa_{33} & -\varkappa_{34} \\
-\varkappa_{41} & -\varkappa_{42} & -\varkappa_{43} & 2 D-\varkappa_{44}
\end{array}\right) \times\left(\begin{array}{c}
\dot{\eta}_{1 \varepsilon_{l}} \\
\dot{\eta}_{2 \varepsilon_{l}} \\
\dot{\eta}_{3 \varepsilon_{l}} \\
\dot{\eta}_{4 \varepsilon_{l}}
\end{array}\right)=\left(\begin{array}{c}
\varkappa_{1}^{e_{l}} \\
\varkappa_{2}^{e_{l}} \\
\varkappa_{3}^{e_{l}} \\
\varkappa_{4}^{\varkappa_{l}}
\end{array}\right) .
$$


Here the notations are used:

$$
\begin{aligned}
& \varkappa_{f 1}=\varkappa_{f 11}\left(\varphi_{1}^{+}+\beta \bar{\nu}_{1}^{+}\right)+\varkappa_{f 12}\left(\beta \nu_{2}^{+}+\beta \bar{\nu}_{2}^{+}\right)+\varkappa_{f 13}\left(\varphi_{1}^{-}+\beta \bar{\nu}_{1}^{-}\right)+\varkappa_{f 14} \beta\left(\nu_{2}^{-}+\beta \bar{\nu}_{2}^{-}\right), \\
& \varkappa_{f 2}=\varkappa_{f 12}\left(\varphi_{2}^{+}+\beta \bar{\nu}_{3}^{+}\right)+\varkappa_{f 11}\left(\beta \nu_{2}^{+}+\beta \bar{\nu}_{2}^{-}\right)+\varkappa_{f 14}\left(\varphi_{2}^{-}+\beta \bar{\nu}_{3}^{-}\right)+\varkappa_{f 13}\left(\beta \nu_{2}^{-}+\beta \bar{\nu}_{2}^{+}\right) \text {, } \\
& \varkappa_{f 3}=\varkappa_{f 11}\left(\varphi_{3}^{+}-\beta \bar{\nu}_{1}^{-}\right)+\varkappa_{f 12}\left(\beta \nu_{2}^{+}-\beta \bar{\nu}_{2}^{+}\right)-\varkappa_{f 13}\left(\varphi_{3}^{-}-\beta \bar{\nu}_{1}^{+}\right)-\varkappa_{f 14}\left(\beta \nu_{2}^{-}-\beta \bar{\nu}_{2}^{-}\right) \text {, } \\
& \varkappa_{f 4}=\varkappa_{f 12}\left(\varphi_{4}^{+}-\beta \bar{\nu}_{3}^{-}\right)+\varkappa_{f 11}\left(\beta \nu_{2}^{+}-\beta \bar{\nu}_{2}^{-}\right)-\varkappa_{f 14}\left(\varphi_{4}^{-}-\beta \bar{\nu}_{3}^{+}\right)-\varkappa_{f 13}\left(\beta \nu_{2}^{-}-\beta \bar{\nu}_{2}^{+}\right) \text {, } \\
& \varphi_{1,3}^{ \pm}=\frac{1}{1-\eta_{1,3}^{2}}+\beta \nu_{1}^{ \pm}, \quad \quad \varphi_{2,4}^{ \pm}=\frac{1}{1-\eta_{2,4}^{2}}+\beta \nu_{3}^{ \pm}, \\
& \nu_{l}^{ \pm}=\nu_{l}^{0 \pm}+\left(\sum_{i=1}^{3} \psi_{l i}^{ \pm} \varepsilon_{i}+\psi_{l 5}^{ \pm} \varepsilon_{5}\right), \quad \bar{\nu}_{l}^{ \pm}=\psi_{l 4}^{ \pm} \varepsilon_{4}+\psi_{l 6}^{ \pm} \varepsilon_{6}, \\
& \nu_{1}^{0 \pm}=\frac{1}{4}\left(J_{11}^{0} \pm J_{13}^{0}\right) \\
& \psi_{1 i}^{ \pm}=\frac{1}{4}\left(\psi_{11 i} \pm \psi_{13 i}\right) \\
& \nu_{2}^{0 \pm}=\frac{1}{4}\left(J_{12}^{0} \pm J_{14}^{0}\right), \quad \nu_{3}^{0 \pm}=\frac{1}{4}\left(J_{22}^{0} \pm J_{24}^{0}\right), \\
& \psi_{2 i}^{ \pm}=\frac{1}{4}\left(\psi_{12 i} \pm \psi_{14 i}\right), \quad \psi_{3 i}^{ \pm}=\frac{1}{4}\left(\psi_{22 i} \pm \psi_{24 i}\right), \\
& \varkappa_{\frac{1}{3} 11}=\left(l_{1+3}^{c}+l_{5+6}^{c}\right)-\eta_{\frac{1}{3}}\left(l_{1+3}^{s}+l_{5+6}^{s}\right), \\
& \varkappa_{\frac{1}{3} 12}=\left(l_{1-3}^{c} \mp l_{7-8}^{c}\right)-\eta_{\frac{1}{3}}\left(l_{1-3}^{s}+l_{7+8}^{s}\right) \text {, } \\
& \varkappa_{\frac{1}{3} 13}= \pm\left(l_{2+4}^{c}+l_{7+8}^{c}\right)-\eta_{\frac{1}{3}}\left(l_{2+4}^{s}-l_{7-8}^{s}\right) \text {, } \\
& \varkappa_{\frac{2}{4} 11}=\left(l_{1-3}^{c} \mp l_{5-6}^{c}\right)-\eta_{\frac{2}{4}}\left(l_{1+3}^{s}+l_{5+6}^{s}\right) \text {, } \\
& \varkappa_{\frac{1}{3} 14}=\left( \pm l_{2-4}^{c}-l_{5-6}^{c}\right)-\eta_{\frac{1}{3}}\left(l_{2-4}^{s}-l_{5-6}^{s}\right) \text {, } \\
& \varkappa_{\frac{2}{4} 13}=\left( \pm l_{2-4}^{c}-l_{7-8}^{c}\right)-\eta_{\frac{2}{4}}\left(l_{2+4}^{s}-l_{7-8}^{s}\right) \text {, } \\
& \varkappa_{\frac{2}{4} 12}=\left(l_{1+3}^{c}+l_{7+8}^{c}\right)-\eta_{\frac{2}{4}}\left(l_{1-3}^{s}+l_{7+8}^{s}\right) \text {, } \\
& \varkappa_{\frac{1}{3} 15}=\left(\mp l_{2-4}^{c}+l_{5-6}^{c}\right)-\eta_{\frac{1}{3}}\left(-l_{2-4}^{s}+l_{5-6}^{s}\right) \text {, } \\
& \varkappa_{\frac{2}{4} 14}=\left( \pm l_{2+4}^{c} \pm l_{5+6}^{c}\right)-\eta_{\frac{2}{4}}\left(l_{2-4}^{s}-l_{5-6}^{s}\right) \text {, } \\
& l_{1 \pm 3}^{c}=\cosh n_{1} \pm a^{2} \cosh n_{3} ; \\
& \varkappa_{\frac{2}{4} 15}=\mp\left(l_{2+4}^{c}+l_{5+6}^{c}\right)+\eta_{\frac{2}{4}}\left(-l_{2-4}^{s}+l_{5-6}^{s}\right) \text {, } \\
& l_{1 \pm 3}^{s}=\sinh n_{1} \pm a^{2} \sinh n_{3} ; \\
& l_{5 \pm 6}^{c}=a a_{46} \cosh n_{5} \pm \frac{a}{a_{46}} \cosh n_{6} ; \\
& l_{5 \pm 6}^{s}=a a_{46} \sinh n_{5} \pm \frac{a}{a_{46}} \sinh n_{6} ; \\
& l_{2 \pm 4}^{c}=\cosh n_{2} \pm a^{2} \cosh n_{4} ; \\
& l_{2 \pm 4}^{s}=\sinh n_{2} \pm a^{2} \sinh n_{4} \text {; } \\
& l_{7 \pm 8}^{c}=a a_{46} \cosh n_{7} \pm \frac{a}{a_{46}} \cosh n_{8} ; \\
& l_{7 \pm 8}^{s}=a a_{46} \sinh n_{7} \pm \frac{a}{a_{46}} \sinh n_{8}, \\
& \varkappa_{f}^{e_{l}}=\beta\left(\psi_{1 l}^{+} \varkappa_{f 11}+\psi_{2 l}^{+} \varkappa_{f 12}\right)\left(\eta_{1}+\eta_{3}\right)+\beta\left(\psi_{2 l}^{+} \varkappa_{f 11}+\psi_{3 l}^{+} \varkappa_{f 12}\right)\left(\eta_{2}+\eta_{4}\right) \\
& +\beta\left(\psi_{1 l}^{-} \varkappa_{f 13}+\psi_{2 l}^{-} \varkappa_{f 14}\right)\left(\eta_{1}-\eta_{3}\right)+\beta\left(\psi_{2 l}^{-} \varkappa_{f 13}+\psi_{3 l}^{-} \varkappa_{f 14}\right)\left(\eta_{2}-\eta_{4}\right)+2 \beta \delta_{l}\left(\rho_{f 1}+\rho_{f 2}\right), \\
& \rho_{\frac{1}{3} 2}=-l_{5+6}^{s} \pm l_{7-8}^{s}+\eta_{\frac{1}{3}}\left(l_{5+6}^{c}+l_{7+8}^{c}\right), \quad \rho_{\frac{2}{4} 1}=2\left(l_{3 \pm 4}^{s}+\eta_{\frac{1}{3}} l_{3+4}^{c}\right), \\
& \rho_{\frac{2}{4} 2}= \pm l_{5-6}^{s}-l_{7+8}^{s}+\eta_{\frac{1}{3}}\left(l_{5+6}^{c}+l_{7+8}^{c}\right), \quad \rho_{\frac{1}{3} j}=l_{5+6}^{s} \pm l_{7-8}^{s}+\eta_{\frac{1}{3}}\left(l_{5+6}^{c}-l_{7+8}^{c}\right), \\
& \rho_{\frac{2}{4} j}=\mp l_{5-6}^{s}+l_{7+8}^{s}+\eta_{\frac{2}{4}}\left(l_{5+6}^{c}-l_{7+8}^{c}\right), \quad l_{3 \pm 4}^{s}=a^{2} \operatorname{sh} n_{3} \pm a^{2} \operatorname{sh} n_{4}, \quad l_{3+4}^{c}=a^{2} \operatorname{ch} n_{3}+a^{2} \operatorname{ch} n_{4} .
\end{aligned}
$$

Constants of piezoelectric stress are obtained by differentiation of electric field over strains at constant polarization, and they can be reduced to such form:

$$
h_{1 j}=\frac{e_{1 j}}{\chi_{11}^{\varepsilon}}, \quad h_{3 j}=\frac{e_{3 j}}{\chi_{33}^{\varepsilon}} .
$$

where the expressions for transverse dielectric permittivities $\chi_{11}^{\varepsilon}$ and $\chi_{33}^{\varepsilon}$ is given by [7]:

$$
\begin{aligned}
& \chi_{11}^{\varepsilon}=\chi_{11}^{\varepsilon 0}+\frac{1}{2 v \Delta}\left[\mu_{13}^{x}\left(\Delta_{1}^{\chi a}-\Delta_{3}^{\chi x}\right)-\mu_{24}^{x}\left(\Delta_{2}^{\chi x}-\Delta_{4}^{\chi x}\right)\right], \\
& \chi_{33}^{\varepsilon}=\chi_{33}^{\varepsilon 0}+\frac{1}{2 v \Delta}\left[\mu_{13}^{z}\left(\Delta_{1}^{\chi z}-\Delta_{3}^{\chi z}\right)+\mu_{24}^{c}\left(\Delta_{2}^{\chi z}-\Delta_{4}^{\chi z}\right)\right] .
\end{aligned}
$$


Here such notation are used:

$$
\begin{aligned}
\Delta & =\left|\begin{array}{cccc}
2 D-\varkappa_{11} & -\varkappa_{12} & -\varkappa_{13} & -\varkappa_{14} \\
-\varkappa_{21} & 2 D-\varkappa_{22} & -\varkappa_{23} & -\varkappa_{24} \\
-\varkappa_{31} & -\varkappa_{32} & 2 D-\varkappa_{33} & -\varkappa_{34} \\
-\varkappa_{41} & -\varkappa_{42} & -\varkappa_{43} & 2 D-\varkappa_{44}
\end{array}\right|, \\
\Delta_{1}^{\chi \alpha} & =\left|\begin{array}{cccc}
\varkappa_{1}^{\chi \alpha} & -\varkappa_{12} & -\varkappa_{13} & -\varkappa_{14} \\
\varkappa_{2}^{\chi \alpha} & 2 D-\varkappa_{22} & -\varkappa_{23} & -\varkappa_{24} \\
\varkappa_{3}^{\chi \alpha} & -\varkappa_{32} & 2 D-\varkappa_{33} & -\varkappa_{34} \\
\varkappa_{4}^{\chi \alpha} & -\varkappa_{42} & -\varkappa_{43} & 2 D-\varkappa_{44}
\end{array}\right|, \Delta_{3}^{\chi \alpha}=\left|\begin{array}{cccc}
2 D-\varkappa_{11} & -\varkappa_{12} & \varkappa_{1}^{\chi \alpha} & -\varkappa_{14} \\
-\varkappa_{21} & 2 D-\varkappa_{22} & \varkappa_{2}^{\chi \alpha} & -\varkappa_{24} \\
-\varkappa_{31} & -\varkappa_{32} & \varkappa_{3}^{\chi \alpha} & -\varkappa_{4} \\
-\varkappa_{41} & -\varkappa_{42} & \varkappa_{4}^{\chi \alpha} & 2 D-\varkappa_{44}
\end{array}\right|, \\
\Delta_{2}^{\chi \alpha} & =\left|\begin{array}{ccccc}
2 D-\varkappa_{11} & -\varkappa_{12} & -\varkappa_{13} & \varkappa_{1}^{\chi \alpha} \\
-\varkappa_{11} & \varkappa_{1}^{\chi \alpha} & -\varkappa_{13} & -\varkappa_{14} \\
-\varkappa_{21} & \varkappa_{2}^{\chi \alpha} & -\varkappa_{23} & -\varkappa_{24} \\
-\varkappa_{31} & \varkappa_{3}^{\chi \alpha} & 2 D-\varkappa_{33} & -\varkappa_{34} \\
-\varkappa_{41} & \varkappa_{4}^{\chi \alpha} & -\varkappa_{43} & 2 D-\varkappa_{44}
\end{array}\right|, \Delta_{4}^{\chi \alpha}=\left|\begin{array}{cccc}
2 D \\
-\varkappa_{21} & 2 D-\varkappa_{22} & -\varkappa_{23} & \varkappa_{2}^{\chi \alpha} \\
-\varkappa_{31} & -\varkappa_{32} & 2 D-\varkappa_{33} & \varkappa_{2}^{\chi \alpha} \\
-\varkappa_{41} & -\varkappa_{42} & -\varkappa_{43} & \varkappa_{4}^{\chi \alpha}
\end{array}\right|,
\end{aligned}
$$

where

$$
\varkappa_{f}^{\chi x}=\varkappa_{f 13} \beta \mu_{13}^{x}+\varkappa_{f 15} \beta \mu_{24}^{x}, \quad \varkappa_{f}^{\chi y}=\varkappa_{f 11} \beta \mu_{13}^{y}+\varkappa_{f 12} \beta \mu_{24}^{y}, \quad \varkappa_{f}^{\chi z}=\varkappa_{f 13} \beta \mu_{13}^{z}+\varkappa_{f 14} \beta \mu_{24}^{z} .
$$

\section{The results of numerical calculations}

For numerical calculation of dielectric and piezoelectric characteristics of GPI we use the parameters determined in [7] from the condition of agreement of calculated characteristics with experimental data, which are enumerated below.

- The parameter of the short-range interactions $w_{0} / k_{\mathrm{B}}=800 \mathrm{~K}$;

- The parameters of the long-range interactions $\tilde{\nu}_{1}^{0+}=\tilde{\nu}_{2}^{0+}=\tilde{\nu}_{3}^{0+}=3.065 \mathrm{~K}, \tilde{\nu}_{1}^{0-}=\tilde{\nu}_{2}^{0-}=\tilde{\nu}_{3}^{0-}=0.05 \mathrm{~K}$, where $\tilde{\nu}_{f}^{0 \pm}=\nu_{f}^{0 \pm} / k_{\mathrm{B}}$.

- The optimal values of the strain potentials: $\tilde{\delta}_{1}=500 \mathrm{~K}, \tilde{\delta}_{2}=600 \mathrm{~K}, \tilde{\delta}_{3}=500 \mathrm{~K}, \tilde{\delta}_{4}=150 \mathrm{~K}, \tilde{\delta}_{5}=100 \mathrm{~K}, \tilde{\delta}_{6}=150 \mathrm{~K} ; \tilde{\delta}_{i}=\delta_{i} / k_{\mathrm{B}} ;$ $\tilde{\psi}_{f 1}^{+}=93.6 \mathrm{~K}, \tilde{\psi}_{f 2}^{+}=252.5 \mathrm{~K}, \tilde{\psi}_{f 3}^{+}=110.7 \mathrm{~K}, \tilde{\psi}_{f 4}^{+}=\tilde{\psi}_{f 6}^{+}=\tilde{\psi}_{f 4}^{-}=\tilde{\psi}_{f 6}^{-}=79.5 \mathrm{~K}, \tilde{\psi}_{f 5}^{+}=22.7 \mathrm{~K}$, $\tilde{\psi}_{f 1}^{-}=\tilde{\psi}_{f 2}^{-}=\tilde{\psi}_{f 3}^{-}=\tilde{\psi}_{f 5}^{-}=0 \mathrm{~K}$, where $\tilde{\psi}_{f i}^{ \pm}=\psi_{f i}^{ \pm} / k_{\mathrm{B}}$.

- The components of effective dipole moments in paraelectric phase are equal to $\mu_{13}^{x}=0.4 \cdot 10^{-18} \mathrm{esu} \cdot \mathrm{cm} ; \mu_{13}^{y}=4.05 \cdot 10^{-18} \mathrm{esu} \cdot \mathrm{cm} ; \mu_{13}^{z}=4.2 \cdot 10^{-18} \mathrm{esu} \cdot \mathrm{cm} ;$ $\mu_{24}^{x}=2.3 \cdot 10^{-18} \mathrm{esu} \cdot \mathrm{cm} ; \mu_{24}^{y}=3.0 \cdot 10^{-18} \mathrm{esu} \cdot \mathrm{cm} ; \mu_{24}^{z}=2.2 \cdot 10^{-18} \mathrm{esu} \cdot \mathrm{cm}$.

In the ferroelectric phase the $y$-component of the first dipole moment is $\mu_{13 \mathrm{ferro}}^{y}=3.82 \cdot 10^{-18} \mathrm{esu} \cdot \mathrm{cm}$, and other components are such as in the paraelectric phase.

- The volume of primitive cell of GPI: $v=0.601 \cdot 10^{-21} \mathrm{~cm}^{3}$.

- The "seed" coefficients of piezoelectric stress $e_{i j}^{0}$, "seed" dielectric susceptibilities $\chi_{i j}^{\varepsilon 0}$ and "seed" elastic constants $c_{i j}^{E 0}$ are obtained as follow:

$e_{i j}^{0}=0.0 \frac{\mathrm{esu}}{\mathrm{cm}^{2}} ; \chi_{11}^{\varepsilon 0}=0.1, \chi_{22}^{\varepsilon 0}=0.403, \chi_{33}^{\varepsilon 0}=0.5, \chi_{31}^{\varepsilon 0}=0.0 ; c_{11}^{0 E}=269.1 \mathrm{kbar}, c_{12}^{E 0}=145 \mathrm{kbar}$,

$c_{13}^{E 0}=116.4 \mathrm{kbar}, c_{15}^{E 0}=39.1 \mathrm{kbar}, c_{22}^{E 0}=\left(649.9-0.4\left(T-T_{\mathrm{c}}\right)\right) \mathrm{kbar}$,

$c_{23}^{E 0}=203.8 \mathrm{kbar}, c_{25}^{E 0}=56.4 \mathrm{kbar}, c_{33}^{E 0}=244.1 \mathrm{kbar}, c_{35}^{E 0}=-28.4 \mathrm{kbar}$,

$c_{55}^{E 0}=85.4 \mathrm{kbar}, c_{44}^{E 0}=153.1 \mathrm{kbar}, c_{46}^{E 0}=-11 \mathrm{kbar}, c_{66}^{E 0}=118.8 \mathrm{kbar}$.

Now let us dwell on the obtained results. The temperature dependences of the coefficients of piezoelectric stress $e_{1 j}, e_{3 j}$ and constants of piezoelectric stress $h_{1 j}, h_{3 j}$ are presented in Fig. 2. These coefficients are equal to zero in the paraelectric phase, because there exists an inversion center. In the ferroelectric phase the coefficients $e_{1 j}$ and $e_{3 j}$, unlike $e_{2 l}$, do not go to infinity at $T \rightarrow T_{c}$; they together with the coefficients $h_{1 j}, h_{3 j}$ slightly change and go to zero at the $T_{\mathrm{c}}$ point. 

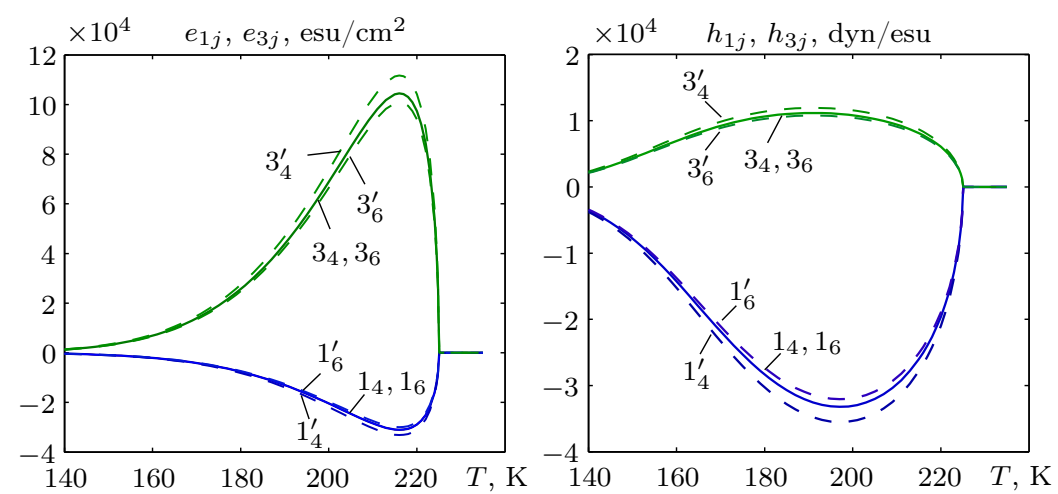

Fig. 2. The temperature dependences of the piezoelectric coefficients $e_{1 j}$ (curves $\left.1_{j}, 1_{j}^{\prime}\right), e_{3 j}\left(3_{j}, 3_{j}^{\prime}\right), h_{1 j}\left(1_{j}, 1_{j}^{\prime}\right)$ and $h_{3 j}\left(3_{j}, 3_{j}^{\prime}\right)$ of GPI crystal. The curves $1_{j}, 3_{j}$ are calculated at $\tilde{\psi}_{f 4}^{+}=\tilde{\psi}_{f 6}^{+}=140 \mathrm{~K}$ and the curves $1_{j}^{\prime}, 3_{j}^{\prime}$ - at $\tilde{\psi}_{f 4}^{+}=150 \mathrm{~K}, \tilde{\psi}_{f 6}^{+}=130 \mathrm{~K}$.

At the low temperatures they also go to zero, because the order parameters $\eta_{f} \rightarrow 1$ and weakly react on the lattice strains. As one can see from (9) and (10), the contributions to the coefficients $e_{1 j}$ from the pseudospins 1 and 2 partially compensate each other, whereas in the case of $e_{3 j}$ they supplement each other. Consequently, the coefficients $e_{3 j}$ have larger values than $e_{1 j}$. Besides, the coefficients $e_{1 j}$ are negative, because the effective dipole moment of the second pseudospin $\mu_{24}^{x}$ is larger than $\mu_{13}^{x}$. However, the coefficients $h_{3 j}$ have smaller values than $h_{1 j}$, because the transverse dielectric permittivity $\chi_{33}^{\varepsilon}$, which is contained in (11), is of one order of magnitude larger than $\chi_{11}^{\varepsilon}$ (see [7]). The values of the coefficients of piezoelectric stress $e_{1 j}$ and constants of piezoelectric stress $h_{1 j}$ depend on the parameters $\tilde{\psi}_{f 4}^{+}$, and the values of $e_{3 j}$ and $h_{3 j}$ depend on the parameters $\tilde{\psi}_{f 6}^{+}$. If these parameters are equal, than $e_{14}=e_{16}, e_{34}=e_{36}, h_{14}=h_{16}, h_{34}=h_{36}$. In the case of different values of $\tilde{\psi}_{f 4}^{+}$and $\tilde{\psi}_{f 6}^{+}$, the values of respective piezoelectric coefficients are different (dashed lines in Fig. 2). The final set of parameters $\tilde{\psi}_{f 4}^{+}$and $\tilde{\psi}_{f 6}^{+}$will be possible after carrying out of experimental measurements of the temperature dependences of $e_{1 j}, e_{3 j}$ and $h_{1 j}, h_{3 j}$.

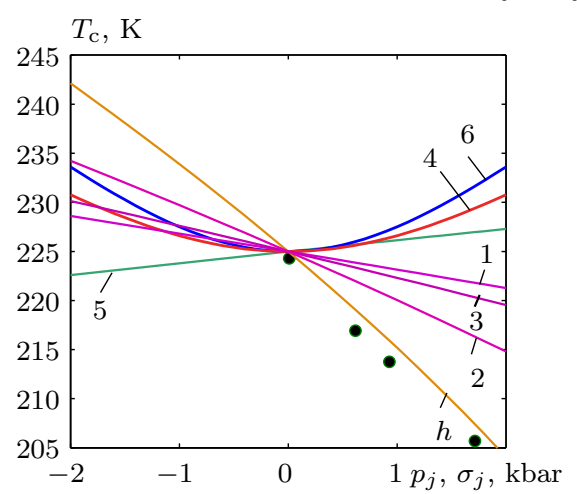

Fig. 3. Dependences of the phase transition temperature $T_{\mathrm{c}}$ of GPI crystal on hydrostatic pressure (curve $h$ ), uniaxial pressures $p_{1}(1), p_{2}(2), p_{3}(3)$ and shear stresses $\sigma_{4}(4), \sigma_{5}(5), \sigma_{6}(6)[7]$. Symbols ' $\bullet$ ' are experimental data [8].

Now let us consider the influence of mechanical stresses on the piezoelectric coefficients. The hydrostatic pressure $p_{i}$, uniaxial pressures $p_{1}, p_{2}, p_{3}$ and shear stress $\sigma_{5}$ do not change the symmetry of GPI crystal. As was determined in [7], application of these pressures leads to weakening of the interactions between pseudospins, in consequence of this the phase transition temperature $T_{c}$ linearly decreases with pressure (see Fig. 3, curves $h, 1,2,3)$. The shear stress $\sigma_{5}$, on the contrary, strengthens interactions and increases the temperature $T_{\mathrm{c}}$ (see Fig. 3, curve 5), This leads to shift of the curves $e_{1 j}(T), e_{3 j}(T), h_{1 j}(T)$ and $h_{3 j}(T)$ to the lower temperatures under pressures and to the higher temperatures under stress $\sigma_{5}$ (see Fig. 4, curves $h$, $1,2,3,5)$. Besides, at the constant deviation of temperature from the phase transition point $\Delta T=T-T_{\rfloor}=-3 \mathrm{~K}$ the piezoelectric coefficients $e_{1 j}(T), e_{3 j}(T), h_{1 j}(T), h_{3 j}(T)$ linearly increase with pressures and linearly decrease with stress $\sigma_{5}$ (see Fig. 5, curves $h, 1,2,3,5)$. It is caused by faster weakening of long-range interactions in comparison with short-range interactions under pressures and by faster strengthening of long-range interactions in comparison with short-range interactions under shear stress $\sigma_{5}$. It should be noted that among the considered above pressures and stress the strongest effect is the effect of hydrostatic pressure $p_{h}$, and the weakest one is the effect of shear stress $\sigma_{5}$.

In [7] it was determined that the shear stresses $\sigma_{4}, \sigma_{6}$ decrease the symmetry of crystal. As a result, the components of spontaneous polarization $P_{1}$ and $P_{3}$ appears in the plane XZ (see Fig. 6); and the dependence $T_{c}\left(\sigma_{4,6}\right)$ is nearly hyperbolic cosine (see Fig. 3, curves 4, 6). Under nonzero stresses $\sigma_{4}$, $\sigma_{6}$ small changes in strains $d \varepsilon_{4}, d \varepsilon_{6}$ are accompanied by change in temperature $d T_{c}$ and by shift of 

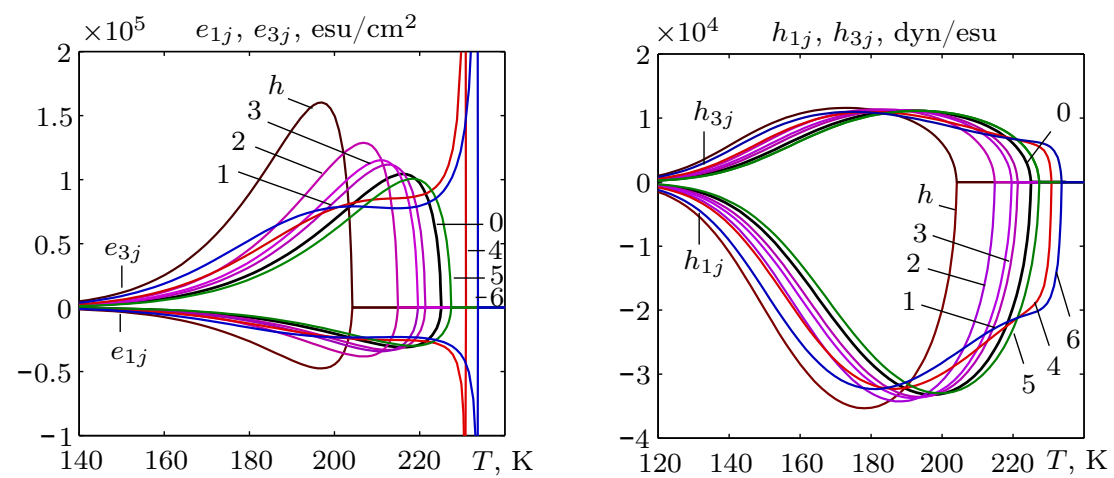

Fig. 4. The temperature dependences of the piezoelectric coefficients $e_{1 j}, e_{3 j}, h_{1 j}$ and $h_{3 j}$ of GPI crystal at the absence of any pressures and stresses (curve 0 ), under hydrostatic pressure (curve $h$ ), uniaxial pressures $p_{1}(1), p_{2}(2), p_{3}(3)$ and shear stresses $\sigma_{4}(4), \sigma_{5}(5), \sigma_{6}(6)$. The value of the pressures is equal to $2 \mathrm{kbar}$.
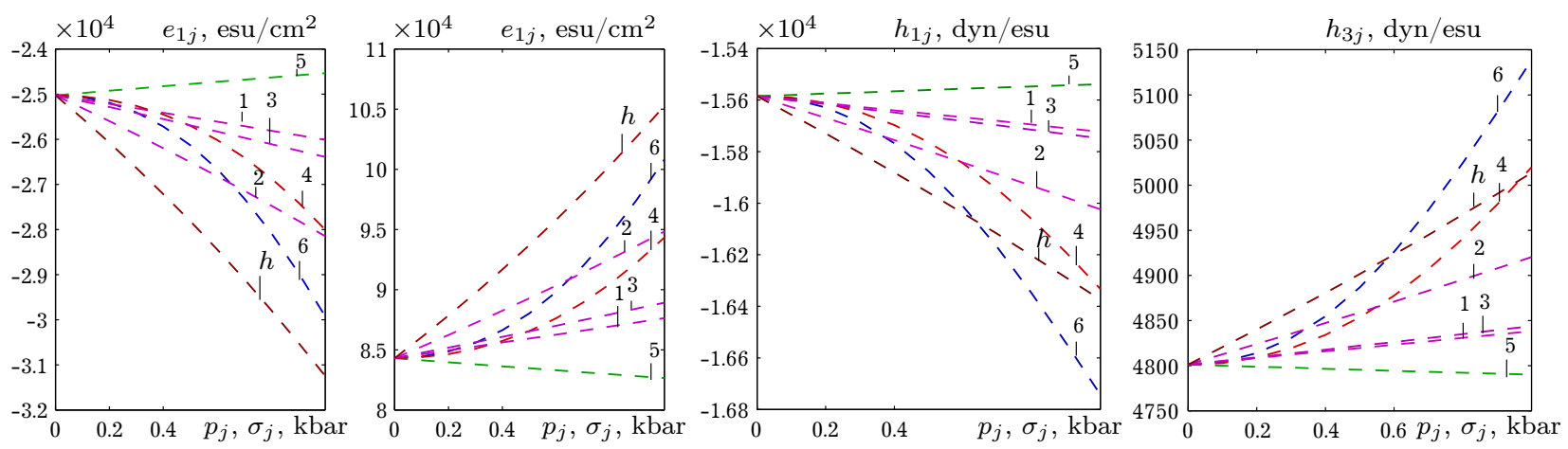

Fig. 5. Dependences of the piezoelectric coefficients $e_{1 j}, e_{3 j}, h_{1 j}$ and $h_{3 j}$ of GPI crystal on hydrostatic pressure (curve $h$ ), uniaxial pressures $p_{1}(1), p_{2}(2), p_{3}(3)$ and shear stresses $\sigma_{4}(4), \sigma_{5}(5), \sigma_{6}(6)$ at the deviation of temperature $\Delta T=-3 \mathrm{~K}$.
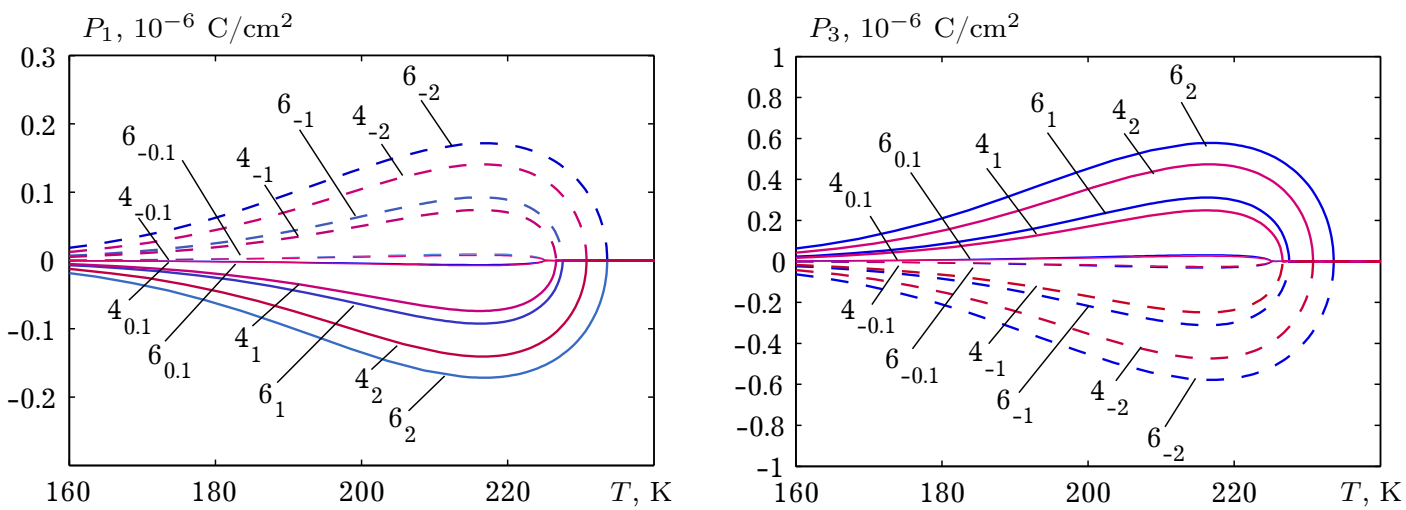

Fig. 6. The temperature dependences of polarizations $P_{1}$ and $P_{3}$ of GPI crystal at different stresses $\sigma_{4,6}$ [7]. Numbers of lines 4 and 6 mean the direction of applied stress $\sigma_{4}$ and $\sigma_{6}$ respectively, the inferior index shows value of the stresses (kbar).

the curves $P_{1}(T)$ and $P_{3}(T)$ to higher temperatures. Since $d P_{i} / d T \rightarrow \infty$ near the phase transition temperature, then $d P_{i} / d \varepsilon_{4} \rightarrow \infty, d P_{i} / d \varepsilon_{6} \rightarrow \infty$. As a result, the temperature dependences $e_{1 j}(T)$, $e_{3 j}(T)$ diverge in the $T_{c}$ point (see Fig. 4 , curves 4,6$)$, and the dependences $e_{1 j}(T), e_{3 j}(T)$ on the stresses $\sigma_{4}, \sigma_{6}$ at constant $\Delta T=-3 K$ are nonlinearly increasing in magnitude (see Fig. 5, curves 4, 6). The piezoelectric coefficients $h_{1 j}, h_{3 j}$ are finite, because as was shown in [7], the transverse components of susceptibility $\chi_{11}^{\varepsilon}$ and $\chi_{33}^{\varepsilon}$, which appear in (11), also diverge in the $T_{c}$ point. 
Now let us consider the influence of transverse electric fields $E_{1}$ and $E_{3}$ on the piezoelectric coefficients. As was shown in [6], these fields decrease the temperature $T_{\mathrm{c}}$ proportionally to $E_{1}^{2}$ and $E_{3}^{2}$, respectively. In Fig. 7 there are presented the temperature dependences of the piezoelectric coefficients
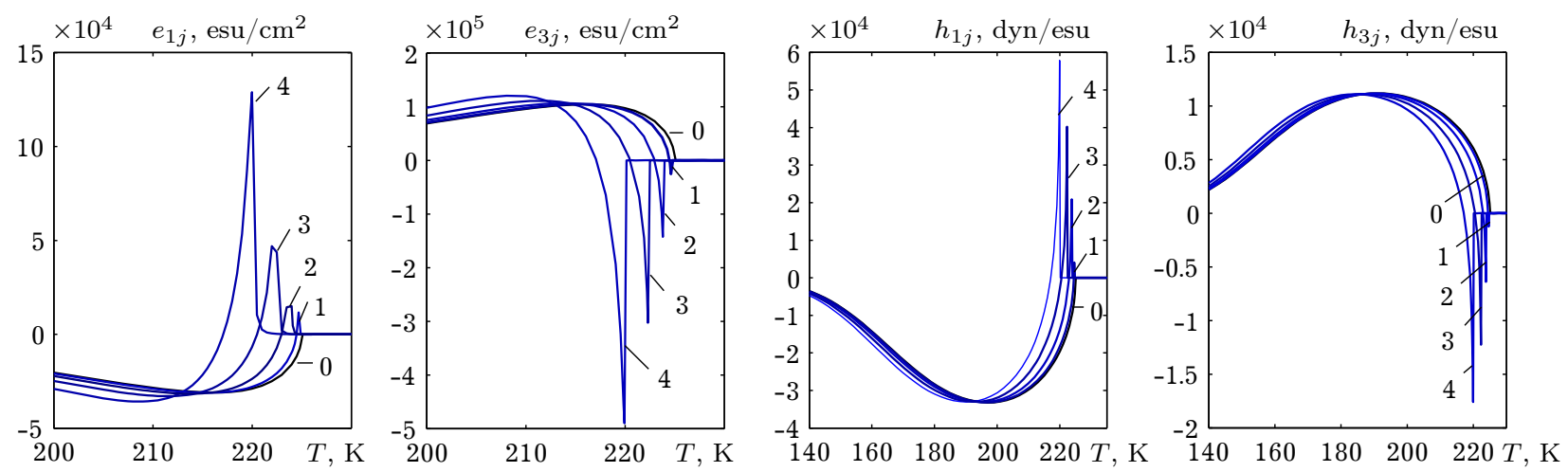

Fig. 7. The temperature dependences of the piezoelectric coefficients $e_{1 j}, e_{3 j}, h_{1 j}$ and $h_{3 j}$ of GPI crystal at different values of the electric field $E_{3}(\mathrm{MV} / \mathrm{m}): 0.0-0 ; 1.0-1 ; 2.0-2 ; 3.0-3 ; 4.0-4$.

$e_{1 j}, e_{3 j}, h_{1 j}$ and $h_{3 j}$ of GPI crystal at different values of the transverse electric field $E_{3}$. Under the influence of field $E_{3}$ in paraelectric phase the centre of inversion disappears, but reflection plane $a c$ remains. Therefore the polarizations of both sublattices (the chains "A" and "B" in Fig. 1) are equal in direction and modulus. At small shear strain $\varepsilon_{4}$ or $\varepsilon_{6}$ the interactions between pseudospins in the chain " $\mathrm{A}$ " become stronger, but in the chain "B" they become weaker. The nascent changes in both sublattice polarizations mutually compensate each other, and the total polarization in the plane $a c$ does not change. That is why the coefficients $e_{1 j}, e_{3 j}, h_{1 j}$ and $h_{3 j}$ are equal to zero.

In the ferroelectric phase the chains " $\mathrm{A}$ " and "B" are ordered in the opposite directions. Therefore the weakening of interactions in the chain " $\mathrm{B}$ " in the presence of the stress $\varepsilon_{4}$ or $\varepsilon_{6}$ leads to the same sign of the changes in polarizations of sublattices. As a result, the piezoelectric coefficients are nonzero.
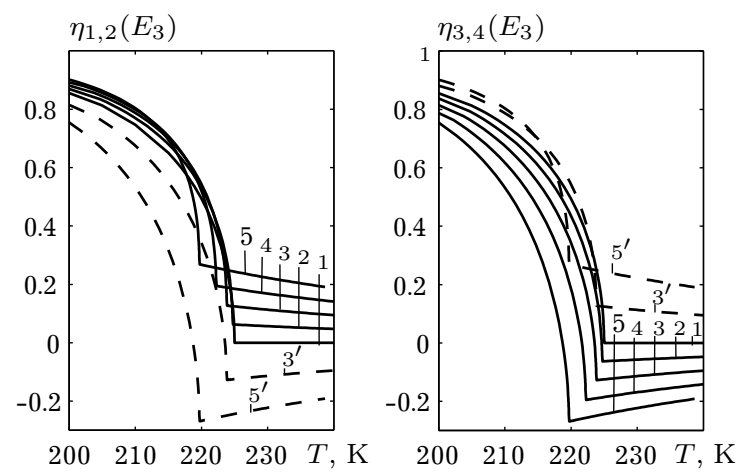

As was shown in [6], in the presence of electric field $E_{3}$ the order parameters $\eta_{3}, \eta_{4}$ decrease stronger than the order parameters $\eta_{1}, \eta_{2}$ increase (see Fig. 8). In result, the chain "B" becomes more sensitive to the strains and external fields in comparison with the chain "A" and in comparison with the case of zero field. At the low temperatures, when the order parameters are close to saturation, the strains $\varepsilon_{4}$ or $\varepsilon_{6}$ additionally weakening interactions in the chain "B". Then the expressions $\dot{\eta}_{3 \varepsilon_{l}}, \dot{\eta}_{4 \varepsilon_{l}}$, which appear in (10), are negative and larger in magnitude than at zero field. That is why the piezoelectric co-

Fig. 8. The temperature dependences of the order parameters $\eta_{f}$ of the GPI crystal at different values of the electric field $E_{3}(\mathrm{MV} / \mathrm{m}): 0.0-1 ; 1.0-2$; $2.0-3 ; 3.0-4 ; 4.0-5 ;-2.0-3^{\prime} ;-4.0-5^{\prime}$.

creasing of electric field strength $E_{3}$. efficients $e_{1 j}, e_{3 j}, h_{1 j}$ and $h_{3 j}$ increase in magnitude at the temperatures far from $T_{\mathrm{c}}\left(T<210 \mathrm{~K}\right.$ for $e_{1 j}$, $e_{3 j}$ and $T<180 \mathrm{~K}$ for $h_{1 j}, h_{3 j}$, in Fig. 7$)$ with in-

At the temperatures close to $T_{\mathrm{c}}$ in the presence of field $E_{3}$ the protons in the chain "B" are almost fully disordered. In the presence of shear stresses the ordering in the chain " $\mathrm{A}$ " increases. Interchain interactions leads to increasing of antiparallel ordering in the chain "B". Consequently, the increment of polarization of the chain "B" appears, which is opposite in sign to the increment of polarization of the chain "A", and larger in magnitude. In result, the piezoelectric coefficients $e_{1 j}, e_{3 j}, h_{1 j}$ and $h_{3 j}$ decrease with strengthening of field, and there appears the negative peak near $T_{\mathrm{c}}\left(T \lesssim T_{\mathrm{c}}\right.$ in Fig. 7$)$, which is finite in magnitude. 
In the presence of positive field $E_{1}$ and positive shear strains the equilibrium values of the order parameters reverse their signs. Consequently, the coefficients $e_{1 j}, e_{3 j}, h_{1 j}$ and $h_{3 j}$ in the presence of this field also reverse their signs (see Fig. 9). The effect of field $E_{1}$ is similar to the effect of field $E_{3}$, but of one order of magnitude larger.
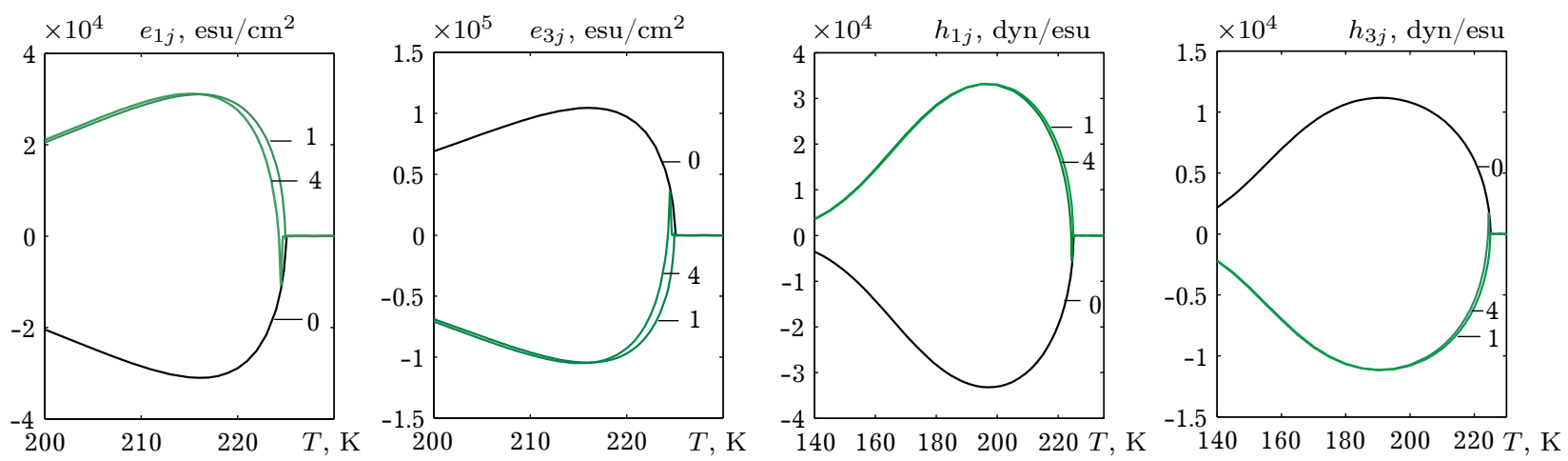

Fig. 9. The temperature dependences of the piezoelectric coefficients $e_{1 j}, e_{3 j}, h_{1 j}$ and $h_{3 j}$ of GPI crystal at different values of the electric field $E_{1}(\mathrm{MV} / \mathrm{m}): 0.0-0 ; 1.0-1 ; 4.0-4$.

Character of changes of the piezoelectric coefficients $e_{1 j}, e_{3 j}, h_{1 j}$ and $h_{3 j}$ with increasing of electric fields $E_{3}$ and $E_{1}$ at the deviations of temperature $\Delta T=-3 \mathrm{~K}$ and $-10 \mathrm{~K}$ is presented in Fig. 10.
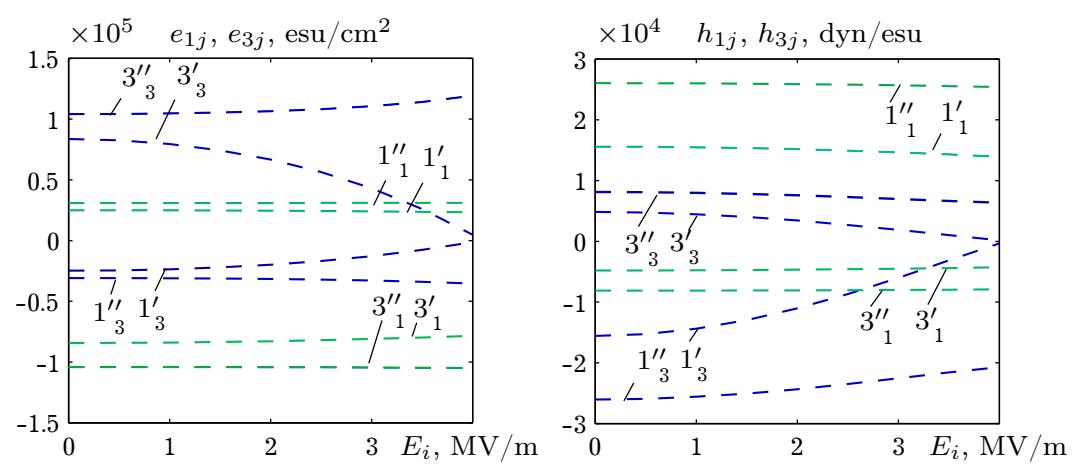

Fig. 10. Dependences of the piezoelectric coefficients $e_{1 j}, e_{3 j}, h_{1 j}$ i $h_{3 j}$ of GPI crystal on electric fields $E_{1}$ and $E_{3}$ at different temperatures. Number of line 1 or 3 means the coefficients $e_{1 j}, h_{1 j}$ or $e_{3 j}, h_{3 j}$, respectively; inferior index 1 or 3 means the external field $E_{1}$ or $E_{3}$, respectively; prime or double prime means the temperature $\Delta T=-3 \mathrm{~K}$ or $-10 \mathrm{~K}$, respectively.

\section{Conclusions}

The transverse piezoelectric coefficients $e_{1 j}$ and $e_{3 j}$, in contrast to the longitudinal coefficients $e_{2 j}$, are finite in the absence of external fields and mechanical stresses. The coefficients $e_{3 j}, h_{3 j}$ are positive but $e_{1 j}, h_{1 j}$ are negative. The hydrostatic $p_{h}$ and uniaxial pressures $p_{1}, p_{2}, p_{3}$, and also shear stress $\sigma_{5}$ change the temperature $T_{\mathrm{c}}$ proportionally to the applied pressures, but qualitatively do not change their behaviour. The shear stresses $\sigma_{4}, \sigma_{6}$ leads to divergence of the coefficients $e_{1 j}, e_{3 j}$ at the temperature $T_{\mathrm{c}}$, but qualitatively do not change the coefficients $h_{1 j}, h_{3 j}$. The transverse electric fields $E_{1}$ and $E_{3}$ at the low temperatures, far from $T_{c}$, increase the piezoelectric coefficients in magnitude, but at the temperatures, close to $T_{\mathrm{c}}$ the finite negative peak appears. In the paraelectric phase they remain equal to zero even in the presence of mechanical stresses or transverse electric fields. Since there are not carried out any experimental measurements of the coefficients $e_{1 j}, e_{3 j}, h_{1 j}$ and $h_{3 j}$, then obtained in this work their temperature, field and pressure dependences for GPI crystal have character of predictions. 
[1] Dacko S., Czapla Z., Baran J., Drozd M. Ferroelectricity in Gly· $\mathrm{H}_{3} \mathrm{PO}_{3}$ crystal. Physics Letters A. 223 (3), 217-220 (1996).

[2] Stasyuk I., Czapla Z., Dacko S., Velychko O. Dielectric anomalies and phase transition in glycinium phosphite crystal under the influence of a transverse electric field. J. Phys.: Condens Matter. 16 (12), 1963 (2004).

[3] ZachekI. R., Shchur Ya, Levitskii R. R., Vdovych A.S. Thermodynamic properties of ferroelectric $\mathrm{NH}_{3} \mathrm{CH}_{2} \mathrm{COOH} \cdot \mathrm{H}_{2} \mathrm{PO}_{3}$ crystal. Physica B. 520, 164-173 (2017).

[4] Zachek I. R., Levitskii R. R., Vdovych A. S. The effect of hydrostatic pressure on thermodynamic characteristics of $\mathrm{NH}_{3} \mathrm{CH}_{2} \mathrm{COOH} \cdot \mathrm{H}_{2} \mathrm{PO}_{3}$ type ferroelectric materials. Condens. Matter Phys. 20 (4), 43707 (2017).

[5] Zachek I. R., Levitskii R. R., Vdovych A. S. The influence of uniaxial pressures on thermodynamic properties of the GPI ferroelectric. J. Phys. Study. 21, 1704 (2017), (in Ukrainian).

[6] Zachek I. R., Levitskii R. R., Vdovych A. S., Stasyuk I. V. Influence of electric fields on thermodynamic properties of GPI ferroelectric. Condens. Matter Phys. 20 (2), 23706 (2017).

[7] Zachek I. R., Levitskii R. R., Vdovych A. S. Deformation effects in glycinium phosphite ferroelectric. Condens. Matter Phys. 21 (3), 33702 (2018).

[8] Yasuda N., Sakurai T., CzaplaZ. Effects of hydrostatic pressure on the paraelectric-ferroelectric phase transition in glycine phosphite $\left(\mathrm{Gly} \cdot \mathrm{H}_{3} \mathrm{PO}_{3}\right)$. J. Phys.: Condens Matter. 9 (33), L347 (1997).

\title{
Розрахунок поперечних п'єзоелектричних характеристик квазіодновимірного сегнетоелектрика фосфіту гліцину
}

\author{
Вдович А. ${ }^{1}$, Зачек I. ${ }^{2}$, Левицький P. ${ }^{1}$ \\ ${ }^{2}$ Інститут фізики конденсованих систем НАН Украӥни, \\ вул. Свенціцьвкого, 1, Лъвів, 79011, Україна \\ ${ }^{1}$ Національний університет "Львівська політехніка", \\ вул. С. Бандери, 12, Львів, 79013, Україна
}

\begin{abstract}
Для дослідження п'єзоелектричних характеристик кристала фосфіту гліцину використано модифіковану модель фосфіту гліцину з урахування п'єзоелектричного зв'язку структурних елементів, які впорядковуються, з деформаціями гратки. В наближенні двочастинкового кластера розраховано поперечні п'єзоелектричні коефіцієнти. Досліджено вплив гідростатичного, одновісних тисків, зсувних напруг і поперечних електричних полів на поперечні п'єзоелектричні характеристики кристала.
\end{abstract}

Ключові слова: сегнетоелектрики, фазовий перехід, n'єзоелектричні коефічієнти, вплив тиску, вплив поля.

2000 MSC: 82D $45,82 \mathrm{~B} 20$

УдК: $536.96,537.226536 .96,537.226(4,82,83,86)$

Mathematical Modeling and Computing, Vol. 5, No. 2, pp. 242-252 (2018) 